\title{
Les absences au travail des femmes suédoises
}

Absence at work of Swedish women

\section{Thierry Ribault}

\section{OpenEdition}

\section{Journals}

Édition électronique

URL : http://journals.openedition.org/travailemploi/4019

DOI : 10.4000/travailemploi.4019

ISSN : 1775-416X

\section{Éditeur}

DARES - Ministère du Travail

\section{Édition imprimée}

Date de publication : 5 décembre 2008

Pagination : 19-32

ISSN : 0224-4365

\section{Référence électronique}

Thierry Ribault, «Les absences au travail des femmes suédoises », Travail et Emploi [En ligne],

116 | octobre-décembre 2008, mis en ligne le 05 novembre 2010, consulté le 19 avril 2019. URL : http://journals.openedition.org/travailemploi/4019; DOI : 10.4000/travailemploi.4019 


\title{
Les absences au travail des femmes suédoises
}

\author{
Thierry Ribault (*)
}

\begin{abstract}
Les taux d'emploi masquent des réalités sociales très disparates selon les pays, ce qui rend hasardeuse leur comparaison immédiate. Les modes de prise en compte des absences, qui renvoient aux politiques de protection sociale en vigueur, ont une influence majeure sur la mesure de ces taux. La Suède présente à ce titre un taux d'absence au travail très élevé, particulièrement chez les femmes. Second constat: la disponibilité temporelle des femmes en Suède exerce un effet structurant sur la nature et les conditions de leur activité professionnelle, en lien notamment avec les conventions qui président à la construction des temps sociaux. Enfin, le cumul d'une charge domestique qui perdure malgré son partage et d'un environnement professionnel dégradé explique pour une partie des femmes en emploi l'ampleur des absences au travail pour cause de maladie. La décommodification de la vie salariale suédoise est un processus non abouti.
\end{abstract}

La Suède présente un taux d'emploi féminin très élevé par rapport à celui en vigueur dans les modèles dits «patricarcaux», qu'ils soient de type méditerranéen comme l'Espagne ou asiatique comme le Japon, ou même par rapport au modèle libéral des États-Unis. En Suède, le recours à la protection sociale pour couvrir les risques professionnels et l'émancipation à l'égard du marché du travail, permettent d'éviter l'exclusion des femmes de ce marché, ce qui contribue à leur taux d'emploi très élevé. A priori le mécanisme inverse semble prévaloir en Espagne ou au Japon, souvent considérés comme des antimodèles sociaux, voire des pays «archaïques» (ADEMA, 2004).

Cette protection sociale, encore appelée « décommodification», ou encore «démarchandisation» renvoie aux activités et aux efforts permettant de réduire l'emprise des forces du marché sur la vie des individus, notamment à travers le welfare state (Esping ANDERsen, 1997). Elle traduit ce qu'est l'objectif fondamental d'un système social: mettre hors du champ du marché un individu, une situation donnée, pour une période de temps définie. La décommodification est généralement mesurée par le taux de remplacement des revenus salariaux par les prestations sociales. Une autre manière de l'apprécier consiste à mettre en relief l'importance relative des absences chez les actifs en emploi. Qu'une société s'organise pour garantir un emploi et un revenu à celles et ceux qui s'absentent de leur travail est un élément dont nous ne discutons ici ni la pertinence, ni le caractère incontestablement progressiste. Ce qui par contre nous amène à nous

\footnotetext{
* CLERSE-Meshs, université Lille 1. E-mail: thierry.ribault@ ifresi.univ-lille1.fr L'auteur remercie les deux rapporteurs anonymes et le comité de rédaction de la revue Travail et Emploi pour leurs critiques constructives.
}

interroger, c'est le poids considérable des absences au travail en Suède, particulièrement celles pour cause de maladie chez les femmes.

L'objectif est donc ici de mettre au jour les mécanismes qui font courir à l'opportunité de progrès social que présente la décommodification, le risque de se transformer en véritable piège, principalement pour des raisons liées à son inachèvement. Nous procédons d'abord à une présentation des statistiques d'absence au travail en Suède et les mettons en perspective internationale. Puis nous examinons en quoi la volonté d'émanciper les femmes suédoises des responsabilités familiales ne les empêche pas de faire face à l'un des emplois du temps domestique les plus chargés, ce qui est en cohérence avec la nature et les conditions d'emploi de ces femmes. Nous explorons ensuite les liens entre d'un côté la double pression - domestique et professionnelle - et de l'autre le poids considérable des absences pour cause de maladie dans l'ensemble des motifs d'absence au travail. En conclusion nous expliquons en quoi on peut parler de décommodification inachevée dans le cas de la Suède, et du piège que cet inachèvement constitue.

\section{Des Suédoises très actives ... et très absentes de leur travail}

En Suède, une femme active occupée sur cinq n'est pas au travail(1), ratio stable depuis 1990. Cette part est de $34 \%$ pour les femmes actives occupées ayant un enfant de moins de 7 ans, $18 \%$

(1) Source: Swedish Labour Force Survey, 2004, Statistics Sweden. Pour 2007, on compte $19 \%$ des femmes actives occupées absentes de leur travail. 
Tableau 1 : Impact des absences sur le taux d'emploi des 16-64 ans (unité : point de taux d'emploi)

\begin{tabular}{|l|c|c|c|c|c|c|c|c|c|c|}
\hline & \multicolumn{2}{|c|}{ Suède } & \multicolumn{2}{c|}{ France } & \multicolumn{2}{c|}{ Espagne } & \multicolumn{2}{c|}{ États-Unis } & \multicolumn{2}{c|}{ Japon } \\
\hline & Hommes & Femmes & Hommes & Femmes & Hommes & Femmes & Hommes & Femmesf & Hommes & Femmes \\
\hline $\begin{array}{l}\text { Maladie, accidents } \\
\text { du travail }\end{array}$ & 2,1 & 3,4 & 1,5 & 1,7 & 1,2 & 0,9 & - & - & - & - \\
\hline Vacances & 6,1 & 6,4 & 6,6 & 5,9 & 1,5 & 1,1 & - & - & - & - \\
\hline $\begin{array}{l}\text { Congé parental, soin } \\
\text { aux enfants malades } \\
\text { et maternité }\end{array}$ & 0,5 & 2,8 & 0,2 & 1,1 & 0,0 & 0,4 & - & - & - & - \\
\hline Formation & 0,3 & 0,5 & 0,2 & 0,1 & 0,0 & 0,0 & - & - & - & - \\
\hline Autres congés & 0,1 & 0,2 & 0,0 & 0,0 & 0,0 & 0,0 & - & - & - & - \\
\hline $\begin{array}{l}\text { Raisons liées au } \\
\text { marché du travail }\end{array}$ & 0,4 & 0,3 & 0,1 & 0,1 & 0,1 & 0,0 & - & - & - & - \\
\hline $\begin{array}{l}\text { Organisation des } \\
\text { horaires }\end{array}$ & 0,8 & 0,7 & 0,1 & 0,1 & 0,0 & 0,0 & - & - & - & - \\
\hline Autres & 0,1 & 0,1 & 0,0 & 0,0 & 0,2 & 0,2 & - & - & - & - \\
\hline Total (a) & 10,3 & 14,3 & 8,6 & 9,0 & 3,0 & 2,7 & 2,2 & 2,7 & 0,9 & 0,9 \\
\hline Taux d'emploi (b) & 75,0 & 71,8 & 68,2 & 56,2 & 75,4 & 49,7 & 69,6 & 56,2 & 80,0 & 58,0 \\
\hline $\begin{array}{l}\text { Taux de présense } \\
\text { En emploi (b-a) }\end{array}$ & 64,7 & 57,5 & 59,6 & 47,1 & 72,4 & 47,0 & 67,4 & 53,5 & 79,1 & 57,0 \\
\hline
\end{tabular}

Source: Labour Force Survey 2004 pour Suède, France, Espagne, Japon, Current Population Survey 2004 pour les États-Unis.

Note: en France la catégorie «congé parental soin aux enfants malades et maternité» correspond à la catégorie «congé non-rémunéré par l'employeur (congé parental, congé sans solde, congé individuel de formation)» d'une part, et à la catégorie «congé de maternité ou de paternité», d'autre part (source Insee, Enquête emploi). En Espagne, la catégorie «congé parental soin aux enfants malades et maternité» correspond essentiellement au congé de maternité.

lorsque l'enfant le plus jeune a entre 7 et 17 ans, et $25 \%$ pour l'ensemble de ces deux populations, c'est-à-dire lorsque l'enfant le plus jeune a moins de 18 ans. Ces chiffres ne recouvrent toutefois que les actifs en emploi qui n'étaient pas présents durant toute la semaine de référence de l'enquête sur l'emploi. Si l'on prend également en considération les actifs occupés n'ayant pas été présents ponctuellement durant la semaine de référence, on aboutit à $37 \%$ de Suédoises actives occupées absentes de leur travail, soit environ 750000 femmes (2).

Ainsi, une fois retirés les absents (au sens restrictif d'absence sur l'ensemble de la semaine de référence de l'enquête) de l'ensemble des actifs occupés, les «taux de présence en emploi» chutent très fortement, notamment pour les femmes, dans les pays qui sécurisent les parcours professionnels: -14 points de taux d'emploi pour la Suède, -9 pour la France contre seulement -3 pour l'Espagne, -3 pour les États-Unis et -1 pour le Japon(3) (cf. tableau 1 et encadré 1).

(2) 734000 hommes sont dans cette situation (source: Labour force Survey 2007).

(3) Nous ne disposons pas pour le Japon des chiffres équivalents à un niveau très détaillé, mais on sait que pour l'ensemble des femmes actives occupées la part d'entre elles absentes du lieu de travail est de moins de $1,6 \%$ en 2003 , et de $1,1 \%$ pour les hommes (source: Annual Report on the Labour Force Survey, résultats détaillés, 2003). En 2006 on obtient respectivement $2 \%$ et $1,5 \%$. Il n'y a pas de système de congé maladie au Japon, hormis les accords contractuels individuels, et les absences de ce type sont généralement prises sur les congés payés (officiellement environ dix jours par an, mais dans la pratique cinq jours sont pris en moyenne) (OGURA, 2004).

\section{Encadré 1}

\section{Les motifs d'absence au travail en Suède et en France}

Dans les deux pays ce sont les vacances qui constituent le motif d'absence le plus important, puis les congés pour maladie ou accident du travail, avec toutefois un impact sur le taux d'emploi de l'absence pour raison de maladie, deux fois plus important dans le cas suédois que dans le cas français: en 2004, le nombre de Suédoises en emploi absentes pour raison de maladie représente 3,4 points de taux d'emploi des femmes (ratio en hausse depuis la fin des années 1990), contre 1,7 points en France. Deuxièmement, la distribution des motifs d'absence entre les deux pays se différencie surtout pour les femmes, alors qu'elle est très proche pour les hommes. Pour ces derniers, on trouve légèrement plus de congés maladie en Suède alors que, comme on l'a vu, on passe du simple au double pour les femmes en Suède par rapport à la France, et on trouve un peu plus de congés de paternité et pour soin aux enfants en Suède, même si ce motif d'absence est très minoritaire dans les deux pays. À ce titre, les spécificités des conventions de genre se reflètent peu dans les statistiques d'absence car, mis à part dans le domaine du congé parental, on observe à peu de chose près les mêmes pratiques dans les deux pays, alors que l'égalité de genre est supposée être plus développée en Suède. Le congé parental, pris comme symbole du régime d'égalité de genre en vigueur en Suède ne représente en fait qu'une petite partie des acquis sociaux tels que l'absentéisme au travail permet de les appréhender. 


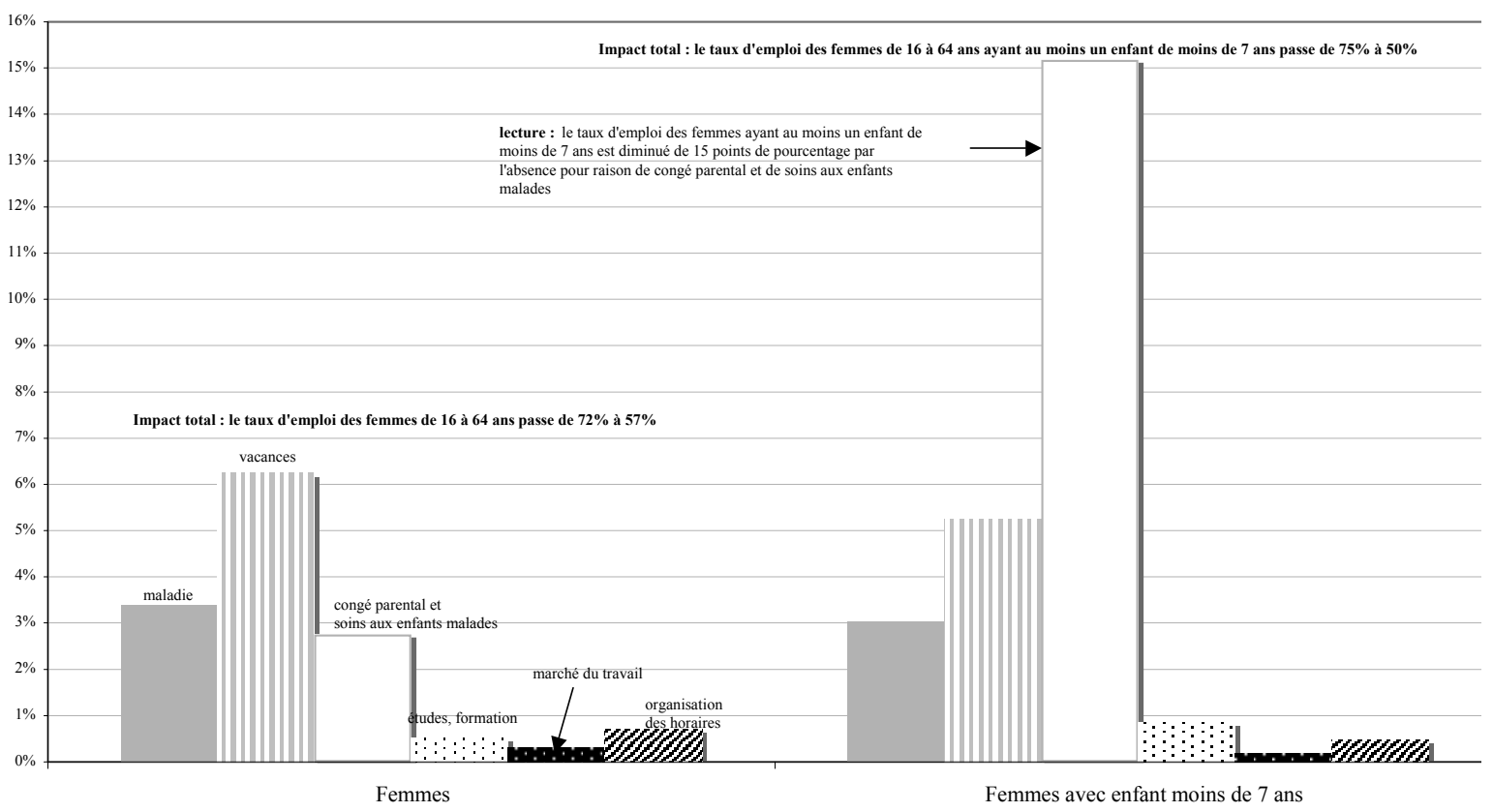

Par conséquent les «taux de présence en emploi», taux d'emploi «ajustés» prenant en considération l'effectivité présentielle du travail rémunéré hors du champs domestique, se retrouvent dans une fourchette qui n'a plus rien à voir avec les écarts initiaux des taux d'emploi qui sont considérables. Selon cette modalité de calcul, le taux d'emploi des Japonaises rejoint même celui des Suédoises. Des estimations identiques menées sur la population des femmes ayant au moins un enfant de moins de 7 ans aboutissent à des résultats allant dans le même sens mais avec un impact encore plus lourd: une baisse de 25 points du taux d'emploi en Suède ramène en effet ce taux de $75 \%$ à $50 \%$ ( $c f$. graphique 1$)$, ce qui le rapproche fortement des taux en vigueur dans nombre de pays dont les institutions sont réputées patriarcales tels que l'Allemagne (57\%), le Royaume-Uni (57\%), l'Italie (53\%), l'Irlande $(52 \%)$, l'Espagne (51\%) ou le Japon (35\%) (4).

Si l'on considère l'ensemble des absents, y compris ceux qui le sont ponctuellement, le taux d'emploi des Suédoises se trouve entamé de 26 points, ce qui le ramène de $72 \%$ à $46 \%$ pour l'année 2006 . Quant aux femmes ayant un enfant de moins de 7 ans, il faudrait pratiquement doubler l'impact de leurs absences sur leur taux d'emploi et le ramener à environ ... $30 \%$.

Donc premier constat: les taux d'emploi masquent des réalités sociales très disparates selon les pays, qui rendent hasardeuse leur comparaison immédiate. Non seulement ces taux incluent de nombreux actifs absents physiquement de leur travail, mais cet absentéisme est lui-même motivé par des raisons multiples et variables selon les pays. Bien que disparates, les

(4) Source: OCDE, 2002. absences ont toutefois un point commun: leur prise en compte constitue un indice du degré de décommodification de la vie salariale dans chaque pays. Les modes de prise en compte des absences, qui renvoient aux politiques de protection sociale, ont en effet une influence majeure sur la mesure des taux d'emploi.

\section{L'effet structurant du poids des tâches domestiques et parentales}

Deuxième caractéristique institutionnellement cohérente avec le constat précédent, la Suède - et dans une moindre mesure la France - tout en ayant le souci d'émanciper les femmes à l'égard du conjoint et des responsabilités familiales (MeULDERS et al., 2007) fait peser sur les femmes une contrainte temporelle domestique plus lourde qu'en Espagne ou au Japon - pays réputés pour leur inégalité de genre - contrainte qui se traduit dans les budgetstemps domestiques des femmes, particulièrement favorables au temps parental.

\section{La priorité suédoise donnée aux enfants}

D'après les données comparatives ( $c f$. encadré 2 pour les sources et les catégories) les femmes suédoises actives consacrent plus de temps aux tâches domestiques et parentales que leurs homologues allemandes, espagnoles, américaines ou japonaises, autant de pays qui ne brillent pourtant ni par leurs politiques d'égalité de genre ni par leurs politiques sociales dans le domaine de la petite enfance.

Une comparaison détaillée montre que le temps de travail domestique des Suédoises actives - et dans 
une moindre mesure celui des Américaines et des Françaises - est nettement plus important que celui des Japonaises, pourtant réputées évoluer dans une société profondément inégalitaire du point de vue du genre: respectivement 28 heures, 27 heures et 31 heures, contre un peu plus de 21 heures au Japon (23 heures en réintroduisant les temps de transport liés à ces tâches). Pour les femmes actives vivant en couple et dont le plus jeune enfant a entre 7 et 17 ans, la Suède affiche un temps domestique supérieur à celui de la France et des Etats-Unis, tandis que celui des Japonaises lui est légèrement supérieur. Mais la Suède présente un temps maternel consacré aux enfants cinq fois plus important qu'au Japon, supérieur de $50 \%$ au temps que leur consacrent les mères Françaises, et de $5 \%$ à celui des Américaines. Lorsque le plus jeune enfant a moins de 7 ans, l'écart de temps parental est confirmé entre la Suède et ses trois partenaires ici considérés : il est de l'ordre de $25 \%$. C'est donc bien l'écart de temps dédié aux enfants qui explique la quasi-totalité de l'écart de temps domestique moyen entre les femmes suédoises, américaines, françaises et japonaises (5). Les femmes suédoises consacrent moins de temps aux tâches proprement ménagères (préparation des repas, nettoyage), mais elles s'investissent beaucoup plus dans les activités liées aux enfants du foyer. Ces dernières représentent, selon l'âge du plus jeune enfant (plus de 7 ans, moins de 7 ans), $19 \%$ à $40 \%$ du temps de travail domestique des Suédoises, contre $3 \%$ à $33 \%$ au Japon, $18 \%$ à $34 \%$ aux États-Unis et $13 \%$ à $38 \%$ en France (tableau 2). En Suède, le passage d'une famille avec enfant(s) en bas âge (moins de 7 ans) à une famille dont le plus jeune enfant a plus de 7 ans, non seulement divise par près de trois le temps consacré aux enfants par les femmes, mais il fait s'accroître de près de $60 \%$ leur temps de travail rémunéré. Les dispositifs de congés parentaux peuvent expliquer ce phénomène. Selon MARC et ZaJdela (2004) «depuis que le congé parental a été instauré, les enfants de moins d'un an sont tous gardés par l'un de leurs parents» (6).

On peut montrer que ces différences de temps domestique et parental ne réflètent en rien des artifices statistiques du type sous-déclaration liée à la simultanéité de certaines activités domestiques (exemple: prendre soin des enfants tout en regardant la télévision), ni un impact des modes de cohabitation, ni encore des effets de structures qui seraient liées aux répartitions des populations actives selon la présence d'un enfant. De fait, les écarts de taux d'emploi féminin entre la Suède et le Japon sont considéra-

(5) Le temps domestique des femmes actives en couple sans enfant est d'ailleurs très proche en Suède et au Japon: respectivement 24 et 23 heures par semaine.

(6) Il s'agit d'une bonne illustration du fait que «nombre des politiques du temps de l'enfant, dans leur concrétisation actuelle, ne participent nullement à une refonte fondamentale de l'organisation du travail et du temps consacré aux jeunes enfants» (Morgan, 2006 p.41).

\section{Encadré 2 \\ Sources utilisées pour les budgets-temps}

Les sources nationales utilisées sont les suivantes. Pour la Suède: Tid för vardagsliv 2001, Statistics Sweden, 2003; pour le Japon: Survey on Time Use and Leisure Activities 2001, Management and Coordination Agency, 2002; pour les États-Unis: American Time Use Survey 2003, Bureau of Labor Statistics, 2004; pour la France: Enquête emploi du temps 1998-1999, Insee, 2000.

On compare ici les emplois du temps des hommes et des femmes ayant entre 20 et 64 ans (ou entre 20 et 84 ans) en Suède, entre 20 et 64 ans (ou 20 ans et plus) au Japon, ayant plus de 18 ans aux États-Unis, et plus de 15 ans en France. Le seuil d'âge du plus jeune enfant est de 6 ans au Japon et aux États-Unis et de 7 ans en Suède et en France.

Les tâches domestiques sont ici définies de la manière suivante: tâches ménagères (cuisiner, mettre la table, nettoyer, laver, coudre, gérer les finances et l'administration, jardinage, entretien de la maison, course), soins aux personnes âgées de la famille, soins aux enfants.

II s'agit de durées moyennes hebdomadaires théoriques, et non de durées par participant effectif à l'activité considérée. Pour le Japon hors temps de transport lié aux tâches domestiques. Pour la France, le temps de transport lié aux loisirs n'est pas distingué de celui lié aux tâches domestiques, ce qui contribue à surévaluer légèrement le temps domestique dans ce pays.

bles: en 2003, ces taux sont respectivement de $80 \%$ et $39 \%$ pour les femmes conjointes dont le plus jeune enfant a moins de 7 ans, et de $89 \%$ et $70 \%$ lorsque le plus jeune enfant a entre 7 et 18 ans (7). Pour autant, l'estimation du temps domestique des femmes japonaises conjointes actives ayant un enfant de moins de 18 ans, sur la base du taux d'activité des Suédoises, n'aboutit qu'à 1 heure de travail domestique hebdomadaire supplémentaire pour les Japonaises, ce qui ne bouscule pas les résultats initiaux.

Ce sont donc plutôt les taux d'emploi qui sont bel et bien «structurés» par les budgets-temps, à travers un certain nombre de conventions dont ils sont un des artefacts: conventions salariales, conventions familiale et de genre, conventions de disponibilité temporelle, conventions de l'enfant également (8).

(7) Source: Swedish Labour Force Survey, 2003; Japan Annual Report on the Labour Force Survey (detailed tabulation), 2003.

(8) La convention de l'enfant a trait à la place et au rôle de l'enfant dans la société et dans la famille. Citons un indicateur comparatif relatif à cette convention au Japon et en Suède: d'après le World Values Survey 2000 (D'AdDIO, Mira D'ErCole, 2005), les femmes des tranches d'âge 15-34 ans et 35-50 ans sont au Japon respectivement $3 \%$ et $5 \%$ à considérer que l'activité des mères est contraire à l'instauration d'une relation chaleureuse et stable avec les enfants, contre $11 \%$ et $12 \%$ en Suède. 
Tableau 2: Temps moyens hebdomadaires par personne

\begin{tabular}{|c|c|c|c|c|c|c|c|c|}
\hline & \multicolumn{2}{|c|}{ Suède } & \multicolumn{2}{|c|}{ Japon } & \multicolumn{2}{|c|}{ États-Unis } & \multicolumn{2}{|c|}{ France } \\
\hline & Femmes & Hommes & Femmes & Hommes & Femmes & Hommes & Femmes & Hommes \\
\hline \multicolumn{9}{|c|}{ Actifs occupés } \\
\hline $\begin{array}{l}\text { Travail domes- } \\
\text { tique et parental }\end{array}$ & 27,9 & 19,8 & 21,5 & 3,1 & 27,0 & 17,2 & 30,7 & 18,1 \\
\hline Travail rémunéré & 32,4 & 41,4 & 38,9 & 54,2 & 34,8 & 44,5 & 35,1 & 44,3 \\
\hline $\begin{array}{l}\text { Soins aux enfants } \\
\text { du foyer }\end{array}$ & 3,6 & 2,2 & 1,5 & 0,5 & 4,0 & 1,9 & 3,0 & 1,3 \\
\hline \multicolumn{9}{|c|}{ Actifs occupés en couple dont le plus jeune enfant a moins de 7 ans } \\
\hline $\begin{array}{l}\text { (1) Travail domes- } \\
\text { tique et parental }\end{array}$ & 41,3 & 26,3 & 39,7 & 5,6 & 38,4 & 22,8 & 35,2 & 17,2 \\
\hline Travail rémunéré & 20,8 & 39,3 & 27,7 & 60,4 & 30,4 & 46,1 & 29,2 & 42,9 \\
\hline $\begin{array}{l}\text { (2) Soins aux } \\
\text { enfants du foyer }\end{array}$ & 16,5 & 8,9 & 13,1 & 2,9 & 13,1 & 6,3 & 13,4 & 4,9 \\
\hline $\begin{array}{l}(2) /(1) \text { En } \% \text { du } \\
\text { temps domestique }\end{array}$ & $40 \%$ & $34 \%$ & $33 \%$ & $52 \%$ & $34 \%$ & $28 \%$ & $38 \%$ & $29 \%$ \\
\hline \multicolumn{9}{|c|}{ Actifs occupés en couple dont le plus jeune enfant a entre 7 et 17 ans } \\
\hline $\begin{array}{l}\text { (1) Travail domes- } \\
\text { tique et parental }\end{array}$ & 31,2 & 21,0 & 32,5 & 2,5 & 29,7 & 18,1 & 30,5 & 14,8 \\
\hline Travail rémunéré & 32,5 & 40,7 & 32,9 & 59,1 & 33,9 & 44,9 & 32,6 & 44,7 \\
\hline $\begin{array}{l}\text { (2) Soins aux } \\
\text { enfants du foyer }\end{array}$ & 5,8 & 3,9 & 1,1 & 0,2 & 5,5 & 2,5 & 3,9 & 1,6 \\
\hline $\begin{array}{l}\text { (2) / (1) En \% du } \\
\text { temps domestique }\end{array}$ & $19 \%$ & $19 \%$ & $3 \%$ & $8 \%$ & $18 \%$ & $14 \%$ & $13 \%$ & $11 \%$ \\
\hline
\end{tabular}

Sources et notes: voir encadré 2.

Le référentiel des politiques sociales et familiales de la Suède (et en partie des autres pays nordiques) est celui du «dual earner-dual carer» (KILKEY, 2003), de la place prépondérante des enfants dans la famille (Letablier, 2003; LALLEMENT, 2002)(9), d'une individualisation et d'une autonomisation croissantes des membres de la société, notamment des femmes et des enfants, et de structures familiales largement dé-nucléarisées. «C'est la pleine individualisation de la société qui est visée et donc la généralisation aux femmes de la pleine citoyenneté.» (DAUNE-RICHARD, Nyberg, 2003, p. 516). L'individualisation des droits sociaux, caractéristique majeure des systèmes de protection sociale des pays nordiques, traduit aussi une difficulté croissante à inscrire les dispositifs de Sécurité sociale dans les réseaux de solidarité familiale: concrètement les grands-parents ne prennent pas en charge leurs petits-enfants, et ils ne sont pas pris en charge par leurs enfants. Pour pallier à ce déficit de solidarité familiale, et en l'absence d'inci-

(9) D'après LaLLement (2002) à la suite d'une réduction significative et comparable de leur temps de travail, les salarié(e)s suédoi(se)s donnent davantage priorité à la famille que leurs équivalents français pour l'usage et la consommation du temps libéré. Selon LetaBLIER (2003 p. 492) «la disponibilité des parents est une valeur importante reconnue par la société comme une condition de l'épanouissement des enfants» et «l'offre d'accueil des enfants a sensiblement augmenté depuis les années 1990 dans les pays nordiques avec pour objectif d'accroître le temps parental consacré aux enfants». tation au recours à la «sous-traitance» de l'activité domestique et parentale, les Suédois et les Suédoises s'absentent du travail, tout en restant en emploi, et leur système de protection sociale les y encourage en limitant les pénalités salariales et professionnelles liées à ces absences.

\section{Préférences ou conventions?}

Selon Anxo, Flood, Kocoglu (2002) etAnxo (2007) les Suédoises consacrent moins de temps aux activités domestiques et parentales et plus de temps aux activités rémunérées que les Françaises (10). Plus souvent actives, les Suédoises seraient en meilleure position sur le marché du travail, du point de vue de leur formation, du salaire, mais aussi des possibilités de modulation des horaires au cours du cycle de vie (congés parentaux, temps partiel choisi). Leur pouvoir de négociation au sein du couple serait ainsi plus grand, et les gains de la spécialisation plus faibles. Cette lecture pose selon nous deux types de problèmes.

D'une part elle mobilise simultanément deux approches contradictoires dans leurs hypothèses. D'un côté, la thèse beckérienne d'une spécialisa-

(10) Les données utilisées par AnXo et al. dans leur analyse descriptive sont celles de 1993 pour la Suède, et il s'agit des classes d'âge de 18-64 ans et des ménages vivant en couple quelle que soit leur situation vis-à-vis de l'emploi. Nos données sont celles de 2001, des classes d'âge de 20-64 ans, pour les actifs occupés. 
tion fondée sur les niveaux de salaire et sur leurs écarts entre hommes et femmes, thèse qui repose sur l'hypothèse d'une répartition des tâches domestiques définie par l'action rationnelle des membres $\mathrm{du}$ foyer, ce qui implique qu'il y a convergence d'intérêt entre les deux époux. D'un autre côté elle mobilise la thèse du pouvoir de négociation et des ressources du pouvoir marital, qui suppose qu'il y a en fait conflit d'intérêt entre les époux et que les ressources à leur disposition (revenu, éducation, statut professionnel) ont une influence considérable sur l'équilibre du pouvoir et sur la négociation dans le foyer (Blood, Wolfe, 1960).

Deuxième problème, lorsqu'on introduit le Japon dans la comparaison, la thèse de la spécialisation perd de sa validité, car les femmes y sont moins spécialisées qu'en Suède ( $c f$. tableau 3 ) alors que l'écart de leur salaire par rapport aux hommes y est plus important. Autrement dit, le constat selon lequel le temps social contraint des Japonaises - qui est la somme de leur temps de travail rémunéré et de leur temps de travail domestique - est plus élevé que pour les Suédoises, et ce, quel que soit l'âge du plus jeune enfant, ne s'explique pas par le poids du temps de travail domestique qui serait plus lourd chez les Japonaises par rapport aux Suédoises, mais bien par un temps de travail rémunéré plus important chez les premières que chez les secondes.

Comme le résume Brossolet (1993) «le modèle de Becker explique la forme la plus répandue d'organisation, celle du ménage conjugal où les conjoints sont spécialisés. La division sexuelle du travail vient donc, selon l'analyse économique, d'un différentiel de productivité entre les hommes et les femmes (ces dernières étant plus productives domestiquement) et d'un souci d'efficacité productive qui conduit à exploiter ce différentiel par la spécialisation (p. 151)». Or nos observations contredisent en partie l'hypothèse beckérienne selon laquelle il serait plus efficient pour le membre du foyer qui est le moins rémunéré (souvent l'épouse) de se spécialiser dans la production domestique, tandis que le membre du foyer le mieux payé (l'époux) se dédie au travail. De fait, la différence de salaire entre genres est plus importante au Japon qu'en Suède, au détriment des femmes, alors que les Japonaises se spécialisent autant voire moins - selon l'âge du plus jeune enfant - dans les tâches domestiques que les femmes suédoises.

Tableau 3 : Degré de spécialisation des hommes et des femmes (temps domestique/temps domestique plus temps de travail rémunéré)

\begin{tabular}{|c|c|c|c|c|}
\hline & Japon & Suède & États-Unis & France \\
\hline Revenu salarial relatif femmes/hommes * & 0,44 & 0,81 & 0,62 & 0,64 \\
\hline \multicolumn{5}{|c|}{ Actifs en couple avec enfant(s) de moins de 18 ans } \\
\hline Homme & $6 \%$ & $37 \%$ & $31 \%$ & nd \\
\hline Femme & $52 \%$ & $57 \%$ & $50 \%$ & $51 \%$ \\
\hline \multicolumn{5}{|c|}{ Actifs en couple avec enfant(s) dont le plus jeune à entre 7 et 17 ans } \\
\hline Homme & $4 \%$ & $34 \%$ & $29 \%$ & $25 \%$ \\
\hline Femme & $50 \%$ & $49 \%$ & $47 \%$ & $48 \%$ \\
\hline \multicolumn{5}{|c|}{ Actifs en couple avec enfant(s) dont le plus jeune à moins de 7 ans } \\
\hline Homme & $8 \%$ & $40 \%$ & $33 \%$ & $29 \%$ \\
\hline Femme & $59 \%$ & $67 \%$ & $56 \%$ & $55 \%$ \\
\hline \multicolumn{5}{|l|}{ Actifs avec ou sans enfants } \\
\hline Homme & $5 \%$ & $32 \%$ & $28 \%$ & $29 \%$ \\
\hline Femme & $36 \%$ & $46 \%$ & $44 \%$ & $47 \%$ \\
\hline \multicolumn{5}{|l|}{ Actifs et inactifs avec ou sans enfants } \\
\hline Homme & $6 \%$ & $36 \%$ & $36 \%$ & $46 \%$ \\
\hline Femme & $53 \%$ & $53 \%$ & $60 \%$ & $70 \%$ \\
\hline
\end{tabular}

Source: voir tableau 2 .

* United Nations Development Fund, Human Development Report, 2006.

Lecture: le temps consacré aux tâches domestiques par les femmes japonaises actives vivant en couple avec un enfant de moins de 18 ans représente $52 \%$ de l'ensemble de leur temps domestique et de leur temps de travail rémunéré.

Quelles que soient les caractéristiques de la population retenue (actifs ou non, avec enfants ou sans), on observe que le poids relatif du temps domestique des hommes est d'autant plus élevé d'un pays à l'autre, que l'inégalité de salaire selon le genre est faible. De ce point de vue, on vérifie donc a priori l'hypothèse beckérienne de spécialisation des hommes dans les tâches où ils sont le mieux payés, à savoir le travail hors de la sphère domestique. Mais pour les femmes, les choses sont plus compliquées. Elles se spécialisent généralement dans les tâches domestiques à un degré 
qui varie selon le niveau d'inégalité salariale qui règne dans chaque pays, mais pour autant ce n'est ni dans le pays où l'inégalité salariale entre genres est la plus élevée, à savoir le Japon, qu'elles se spécialisent le plus dans les tâches domestiques, ni a contrario, dans le pays où les inégalités salariales selon le genre sont les plus faibles, à savoir la Suède, qu'elles se spécialisent le moins. Ceci est particulièrement vrai pour les couples actifs avec enfant(s) de moins de 18 ans.

On sort donc de la logique circulaire que dénonce Brossolet (1993) selon laquelle «la spécialisation dans la famille est expliquée par la position défavorisée des femmes sur le marché du travail, et la division sexuelle du marché, c'est-à-dire cette défaveur des femmes, est elle-même expliquée par la plus grande participation des femmes aux contraintes imposées par la famille [...]». Les femmes japonaises sont en effet relativement moins spécialisées dans les tâches domestiques, comparées aux Suédoises notamment, alors qu'elles sont largement défavorisées sur le marché du travail. Il y a donc bien là à l'œuvre un jeu de conventions qui contribuent à la construction sociale de l'emploi et du non-emploi féminin, du temps de travail et du temps hors travail.

Voyons en quoi ces conventions sont déterminantes dans la nature des activités occupées par les femmes.

\section{Quels emplois pour les femmes en Suède?}

C'est parce que les femmes suédoises assument un volume d'heures de travail domestique important, compte tenu des conventions en vigueur, et malgré le contexte d'égalité de genre auquel elles sont rattachées, qu'elles se trouvent prioritairement disponibles pour des emplois dans les secteurs sociaux qui sont adaptés à leur offre de disponibilité (aménagements d'horaires, temps partiel). Cette adaptation n'exclut cependant pas que ces emplois puissent être vécus comme contraignants par leurs détentrices.

\section{Des métiers de femmes}

Ainsi, dans le cas suédois, le temps «libéré» des contraintes domestiques va prioritairement vers des activités salariées de services sociaux publics, en partie en cohérence avec les conventions d'égalité de genre qui appellent une mise en activité des femmes, mais en partie aussi en cohérence avec les contraintes temporelles que les activités domestiques des femmes font peser sur leur disponibilité professionnelle. Celle-ci est limitée par l'ampleur des tâches domestiques conventionnellement nécessaires, et les emplois publics de services sociaux sont mieux adaptés à une offre de travail contrainte, même si en contrepartie les opportunités de promo- tion y sont plus limitées que dans d'autres secteurs. La persistance d'une ségrégation sexuée du marché du travail suédois parmi les plus élevées des pays occidentaux en est sans doute une des conséquences (Daune-Richard, Nyberg, 2003; Daune-Richard 2004; Wehner, Abrahamson, 2003), puisque ce sont principalement les métiers de soin, d'éducation, de santé et d'aide sociale où l'on retrouve les femmes, professions où elles sont largement surreprésentées. En 2005, plus de la moitié des femmes salariées travaillent dans des structures municipales et étatiques contre $18 \%$ des hommes, la moitié des femmes travaillent dans le secteur social, de la santé et de l'éducation, contre $14 \%$ des hommes. Les femmes représentent $85 \%$ des emplois du secteur social, $83 \%$ de ceux de la santé, $72 \%$ de ceux de l'éducation et $80 \%$ des emplois municipaux qui couvrent partiellement ces secteurs d'activité(11).

Le principal symptôme de la ségrégation professionnelle soutenue en Suède, réside dans le fait qu'une part considérable de la force de travail féminine non agricole est mobilisée dans des métiers «féminins». Ainsi, en 2001, plus d'une femme active sur deux exercent un métier féminisé à plus de $80 \%$, contre plus d'une femme sur trois aux États-Unis ( $c f$. tableau 4). L'écart avec le Japon est considérable puisque dans ce pays seulement $15 \%$ des femmes actives sont dans cette situation. De même, une Suédoise active sur trois et environ une Américaine sur quatre exerce un métier féminisé à plus de $90 \%$, contre seulement $8 \%$ des Japonaises. En outre, dans le cas du Japon, la part des femmes, selon le degré de féminisation des métiers, est stable sur les trois dernières décennies. Le niveau de ségrégation professionnelle selon le genre n'est donc pas nécessairement corrélé positivement à l'inégalité de rémunération selon le genre: la Suède combine un niveau élevé de ségrégation professionnelle et un niveau élevé d'égalité de genre dans la société, tandis qu'au Japon, le niveau relativement bas de ségrégation professionnelle selon le genre s'accommode d'une forte inégalité de genre en général (salariale, responsabilités, vie politique).

\section{Tableau 4 : Part des femmes dans l'emploi non-agricole selon le degré de féminisation des métiers (en \%)}

\begin{tabular}{|l|r|r|r|}
\hline & \multicolumn{1}{|c|}{ Japon } & \multicolumn{1}{|c|}{ États-Unis } & \multicolumn{2}{|c|}{ Suède } \\
\hline $70 \%$ et plus & 29 & 52 & 68 \\
\hline $80 \%$ et plus & 15 & 37 & 56 \\
\hline $90 \%$ et plus & 8 & 24 & 31 \\
\hline
\end{tabular}

Source: Suède: Statistics Sweden, Labour Force Survey 2001, Japon: Recensement de la population 2000; États-Unis: Current population Survey 2002, Bureau of Labour Statistics.

Lecture: $29 \%$ des femmes actives japonaises, $68 \%$ des Suédoises et $52 \%$ des Américaines occupent des métiers féminisés à plus de $70 \%$.

(11) Source: Statistics Sweeden 2008. 
Une des externalités des politiques publiques des pays nordiques qui visent à améliorer l'égalité de genre sur le marché du travail en particulier et dans la société en général, est donc d'exposer les femmes à une ségrégation professionnelle accrue, car les recrutements sur les emplois qui viennent en soutien des tâches domestiques auparavant menées dans le foyer sans rémunération, se font principalement auprès des femmes (12). Mais surtout, fait plus préoccupant, ces emplois ne dispensent pas les femmes suédoises de subir, comme nous allons le voir, des pressions considérables dommageables à leur état de santé.

\section{Des emplois adaptés mais contraignants}

Les métiers les plus féminisés affichent les pourcentages les plus importants d'individus soumis à de fortes pressions professionnelles, c'est-à-dire des métiers pour lesquels l'employeur attend un niveau élevé de performances sans que le salarié ait la possibilité de contrôler et d'influencer son activité: en 2001, les deux tiers des infirmières, $55 \%$ des enseignantes d'écoles primaires, plus de la moitié des enseignantes du secondaire, et des enseignantes de maternelle, $46 \%$ des assistantes hospitalières, $43 \%$ des professionnelles de la petite enfance et $37 \%$ des aides à domicile sont dans cette situation(13). La confrontation des distributions suédoise et japonaise de la mobilisation au travail selon l'heure de la journée est éclairante. Le Japon est un contrepoint intéressant dans la mesure où il constitue le parangon de la société salariale du 24 heures sur 24. Cette confrontation montre non sans surprise que l'atypisme des horaires de mobilisation au travail des femmes actives est plus important chez les Suédoises que chez les Japonaises, particulièrement sur les créneaux de nuit et du matin très tôt, ceci manifestement afin de libérer les heures de l'après-midi, probablement en grande partie au bénéfice des enfants(14). Le caractère particulièrement atypique des périodes de travail des femmes en Suède est confirmé par les comparaisons internationales: la Suède est, avec le Luxembourg, le pays où la surreprésentation des femmes actives occupées régulièrement les week-ends est la plus élevée (Presser, GorNICK, 2005).

Si l'on se concentre sur la tranche d'âge des 2549 ans, c'est-à-dire la période durant laquelle les femmes sont généralement doublement mobilisées,

(12) Ce constat doit être toutefois nuancé. D'une part, les pouvoirs publics ne sont pas les seuls à contribuer à la création d'emplois féminisés, d'autre part, on observe dans les pays nordiques sur les deux dernières décennies un mouvement de réduction de la ségrégation professionnelle dont l'ampleur est bien plus importante que ce que l'on observe dans les autres pays de l'OCDE, à l'exception de l'Amérique du Nord (MELKAS, HANKER, 2003).

(13) Source: Statistics Sweden 2006.

(14) Pour une confirmation plus ancienne de l'importance de l'atypisme des horaires de travail et de son articulation avec la place de l'enfant dans la famille en Suède voir NYSTRÖM, GAUNT (1986). au travail et dans les tâches domestiques et parentales, on constate que la Suède ne se distingue pas de ses partenaires européens en dépit de sa réputation de «conciliateur». La mobilisation des Suédoises de cette tranche d'âge est relativement élevée le dimanche, la nuit ou en soirée, ou encore en travail posté ( $c f$. tableau 5). De même la part des femmes entre 25 et 49 ans qui affirment ne jamais travailler sur ces périodes atypiques est loin de figurer parmi les plus élevées de l'ensemble des pays européens (cf. tableau 5 bis). Ce constat est d'ailleurs également valable concernant les populations de femmes de 15 ans et plus ou sur la tranche des 15-39 ans.

Tableau 5 : Atypisme des périodes de travail des femmes en emploi de 25 à 49 ans en \%

\begin{tabular}{|l|r|r|r|r|r|}
\hline & $\begin{array}{c}\text { Travail } \\
\text { posté }\end{array}$ & Samedi & Dimanche & Nuit & Soirée \\
\hline Belgique & 7,9 & 20,2 & 10,5 & 3,0 & 10,9 \\
\hline Danemark & 5,2 & 17,1 & 13,2 & 3,2 & 13,0 \\
\hline Allemagne & 15,2 & 27,1 & 14,5 & 6,7 & 24,7 \\
\hline Grèce & 17,9 & 30,1 & 8,1 & 2,8 & 22,1 \\
\hline Espagne & 17,5 & 29,4 & 12,8 & 3,8 & 17,3 \\
\hline France & 6,8 & 34,1 & 15,0 & 4,8 & 15,0 \\
\hline Italie & 17,8 & 35,3 & 11,9 & 5,0 & 11,5 \\
\hline Luxembourg & 9,3 & 19,4 & 11,6 & 4,4 & 11,6 \\
\hline Pays-Bas & 7,0 & 24,9 & 17,9 & 7,4 & 27,4 \\
\hline Autriche & 16,8 & 30,7 & 16,8 & 5,1 & 10,9 \\
\hline Portugal & 18,6 & 22,0 & 10,8 & 5,6 & $\mathrm{ND}$ \\
\hline Finlande & 25,9 & 23,0 & 16,2 & 8,1 & 24,0 \\
\hline Suède & 27,5 & 14,5 & 12,3 & 5,0 & 14,5 \\
\hline Royaume-Uni & 16,0 & 17,2 & 10,7 & 9,1 & 23,6 \\
\hline Norvège & 26,9 & 16,3 & 8,6 & 3,5 & 11,3 \\
\hline
\end{tabular}

Source: European Labour Force Survey 2007, Eurostat.

Note: personne affirmant travailler «habituellement» selon les modalités indiquées en \% des femmes de 25 à 49 ans en emploi.

Tableau 5 bis : Atypisme des périodes de travail des femmes en emploi de 25 à 49 ans en \%

\begin{tabular}{|l|r|r|r|r|}
\hline & Samedi & Dimanche & Nuit & Soirée \\
\hline Belgique & 63,5 & 77,2 & 91,5 & 71,4 \\
\hline Danemark & 66,4 & 72,5 & 91,5 & 60,6 \\
\hline Allemagne & 56,1 & 74,0 & 88,9 & 58,4 \\
\hline Grèce & 49,6 & 77,1 & 89,5 & 44,7 \\
\hline Espagne & 65,3 & 83,7 & 91,4 & 72,5 \\
\hline France & 49,0 & 70,3 & 89,7 & 70,4 \\
\hline Italie & 57,5 & 83,1 & 92,8 & 84,3 \\
\hline Luxembourg & 76,2 & 84,6 & 95,0 & 87,2 \\
\hline Pays-Bas & 61,4 & 71,1 & 88,7 & 58,4 \\
\hline Autriche & 57,6 & 75,7 & 85,2 & 71,7 \\
\hline Portugal & 59,7 & 78,4 & 86,9 & $\mathrm{ND}$ \\
\hline Finlande & 67,5 & 76,1 & 87,7 & 56,4 \\
\hline Suède & 67,5 & 70,4 & 88,8 & 67,8 \\
\hline Royaume-Uni & 57,7 & 71,2 & 84,6 & 56,1 \\
\hline Norvège & 62,5 & 74,0 & 86,4 & 61,4 \\
\hline
\end{tabular}

Source: European Labour Force Survey 2007, Eurostat.

Note: personne affirmant ne jamais travailler selon les modalités indiquées en $\%$ des femmes de 25 à 49 ans en emploi. 
Graphique 2. Suède : actifs occupés et absents du travail selon le motif

(unité: 100 personnes ; source: Statistics Sweden, données non disponibles pour 1980 et 1986)

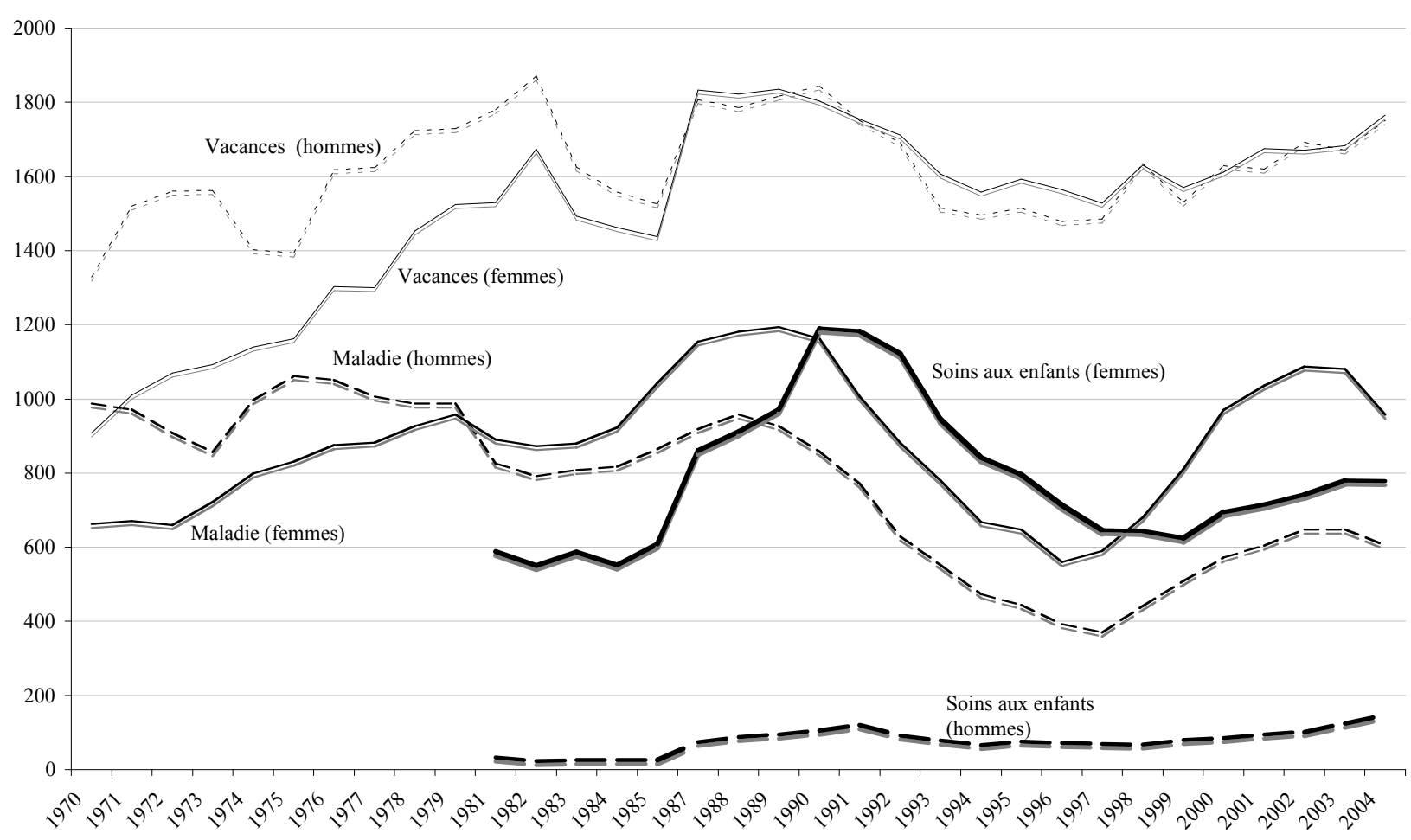

En résumé, la disponibilité temporelle des femmes en Suède exerce un effet structurant sur la nature et les conditions de leur activité professionnelle, en lien notamment avec les conventions qui président à la construction des temps sociaux, temps de travail et temps domestique et parental.

\section{Une forte vulnérabilité sanitaire des Suédoises en emploi}

Voyons maintenant comment le cumul d'une charge domestique qui perdure malgré le partage des tâches domestiques, et d'un environnement professionnel dégradé, explique pour une partie des femmes en emploi l'ampleur de leurs absences au travail pour cause de maladie.

\section{Un nombre considérable d'absences pour maladie}

Le congé parental, tout comme les congés pour soigner un enfant malade (soixante jours par an et par enfant avec une couverture de $85 \%$ du salaire), concerne $19 \%$ de l'ensemble des femmes actives occupées absentes, tandis que la majeure partie (44\%) qui ne travaillait pas durant la semaine de référence de l'enquête est tout simplement en vacances. Mais, plus inquiétant, les arrêts maladie constituent un quart des absences des femmes (15).

Si on ajoute aux 82000 femmes en congé maladie déclarées absentes durant toute la semaine de réfé- rence de l'enquête, celles absentes pour des raisons de maladie mais de manière ponctuelle, on obtient 155000 personnes en 2006 , soit $7,5 \%$ des actives occupées, à comparer aux 4,7 \% (107000 personnes) d'hommes actifs occupés dans la même situation.

Ainsi, si le droit de retrait temporaire du marché du travail pour exercer des responsabilités familiales représente indéniablement une caractéristique forte du système suédois de protection sociale, il ne constitue ni la seule ni la plus importante cause d'absence de la population en emploi dans ce pays. Depuis la fin des années 1990, le congé maladie est beaucoup plus répandu que le congé parental, même chez les femmes ( $c f$. graphique 2).

$\mathrm{Au}$ total en 2006, 6\% des heures travaillées par les femmes en Suède correspondent à des heures d'absence pour maladie contre moins de $4 \%$ en 1996. Alors que parmi les hommes en emploi on observe une baisse tendancielle du nombre d'absences pour maladie depuis 1970, l'évolution est à la hausse chez les femmes.

D'après la Swedish Social Insurance Agency, en 2005, plus de 80000 femmes bénéficiaient d'un congé maladie de plus de 180 jours, contre 45000 pour les hommes. Les bénéficiaires étaient respectivement de 50000 et 40000 dans les années 1990 , et de l'ordre de 20000 et 30000 durant les années 1970 et 1980 . Il est donc peu étonnant que les montants des compensations du revenu d'activité pour cause de maladie aient été multipliés par près de deux entre 2000 et 2006(16). 


\section{L'exception suédoise}

Les données comparatives dont nous disposons rendent compte d'un écart considérable en matière d'absence au travail pour maladie en Suède par rapport aux autres pays européens. Ainsi, en 2005, plus de $37 \%$ des salariées suédoises entre 25 et 49 ans ont été absentes de leur travail pour cause de maladie dans la famille au cours des douze derniers mois précédant l'enquête. Ce pourcentage est parmi les plus élevés d'Europe ( $c f$. tableau 6 ). $66 \%$ de ces personnes absentes pour maladie sont couvertes par un système de congé spécial rémunéré.

Tableau 6 : Part des salariés s'étant absentés du travail au cours des douze derniers mois pour cause de maladie dans la famille en $\%$

\begin{tabular}{|l|c|c|}
\hline & $\begin{array}{c}\text { Part des } \\
\text { absents pour } \\
\text { maladie }\end{array}$ & $\begin{array}{c}\text { Dont part de ceux } \\
\text { bénéficiant } \\
\text { d'un congé spécial } \\
\text { rémunéré }\end{array}$ \\
\hline $\begin{array}{l}\text { Union européenne } \\
\text { (quinze pays) }\end{array}$ & 21,8 & 42,2 \\
\hline Belgique & 14,7 & 48,1 \\
\hline Danemark & 41,5 & 74,2 \\
\hline Allemagne & 8,0 & 31,9 \\
\hline Irlande & 22,4 & 53,2 \\
\hline Grèce & 29,5 & 41,9 \\
\hline Espagne & 23,2 & 52,8 \\
\hline France & 26,9 & 31,5 \\
\hline Italie & 30,0 & 37,4 \\
\hline Luxembourg & - & - \\
\hline Pays-Bas & 2,8 & 44,9 \\
\hline Autriche & 21,8 & 38,5 \\
\hline Portugal & 26,0 & 17,0 \\
\hline Finlande & 25,0 & 64,3 \\
\hline Suède & 37,2 & 65,9 \\
\hline Royaume-Uni & 28,2 & 47,2 \\
\hline Norvège & 37,5 & 45,1 \\
\hline
\end{tabular}

Source: European Labour Force Survey 2005, Eurostat.

C'est aussi en Suède que l'on trouve le nombre de jours ouvrables non-travaillés pour cause de maladie le plus élevé des pays de l'Ocde. En 2004 on comptait vingt-six jours annuels contre seize en France, treize au Royaume-Uni, dix au Danemark, neuf aux ÉtatsUnis, sept en Allemagne (RAE, 2005).

L'Ocde considère de son côté que le recours élevé aux congés pour maladie se fait au détriment d'une utilisation efficace de la force de travail, et regrette que la Suède, la Norvège et la Finlande soient les trois pays de l'Ocde où le nombre moyen de semaines non travaillées pour cause de maladie est le plus élevé: respectivement $2,5,2$ et 1,5 semaines par salarié et par an (OCDE 2007 et 2008). Notre perspective est différente: moins préoccupés par l'état de santé des comptes sociaux que par celui des travailleurs, nous proposons de prendre au sérieux les signaux que constituent les déclarations d'absence au travail pour maladie.

\section{Un état de santé dégradé lié au travail}

Dans un pays qui dispose d'un congé parental relativement généreux il est peu probable que tout ou partie de ce congé maladie soit en fait investi de manière détournée dans le temps parental. Les classes d'âge 35-44 ans et 45-54 ans rassemblant à elles seules respectivement près d'un tiers des absences pour cause de maladie chez les femmes en emploi, l'interprétation la plus vraisemblable renvoie à l'intensification du travail qui génère une montée d'insatisfactions voire de pathologies qui se reflètent dans ces absences nombreuses, une partie de ces pathologies pouvant découler de la double charge de travail - domestique et salariale - que connaissent les femmes suédoises à certaines périodes de leur vie. On a vu plus haut la prégnance des tâches domestiques dans l'emploi du temps des Suédoises. Ce constat relativise aussi l'argument selon lequel les salariés suédois utiliseraient le congé maladie comme moyen de se mettre en préretraite anticipée à partir de 55 ans (RAE, 2005).

RAE (2005) estime que les bénéficiaires suédois des congés maladie ne sont pas dans un système d' «obligations mutuelles » et que leur état de santé, mesuré notamment par l'espérance de vie, ne justifie pas de telles absences. On peut toutefois s'inquiéter du nombre d'études de ce genre, visant à identifier les déterminants de l'absence au travail pour maladie sans prendre en compte, exception faite de l'espérance de vie, les variables liées précisément à l'état de santé des personnes absentes (17)! Non seulement les résultats de ces travaux sont en forte contradiction avec les données que nous fournissent les enquêtes de la European Foundation for the Improvement of Living and Working Conditions, mais elles le sont aussi avec les résultats des recherches socio-sanitaires menées en Suède.

D'après l'enquête européenne sur les conditions de travail de 2005, près du tiers des femmes Suédoises en emploi s'absentent de leur travail pour des raisons de santé liées à leur travail. $12 \%$ de ces femmes absentes durant les douze derniers mois précédant l'enquête l'ont été plus de soixante jours, et $8 \%$ de la même population attribuent plus de soixante jours de leur absence à des problèmes de santé dus à leur travail. Pour chacun de ces indicateurs, la Suède se situe dans la tranche haute parmi les pays européens ( $c f$. tableau 7).

La Suède est, avec la Grèce, un des pays européens où le pourcentage de femmes en emploi déclarant avoir leur état de santé affecté par leur travail est le plus élevé: $57 \%$. Parmi ces femmes, $42 \%$ se déclarent affectées par des troubles musculo-squelettiques, $41 \%$ par le stress, $20 \%$ par des troubles

(17) Voir Bonato, Lusine (2004) comme autre exemple typique de cette démarche et pour une bibliographie très complète de travaux relevant du même esprit. 
du sommeil, $18 \%$ par l'anxiété et $15 \%$ par l'irritabilité. La Suède est un des pays d'Europe où les fréquences de déclaration de ces pathologies par les femmes sont les plus affirmées ( $c f$. tableau 7bis).

Tableau 7 : Indicateurs comparés de santé au travail

\begin{tabular}{|c|c|c|c|}
\hline & 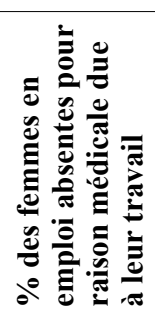 & 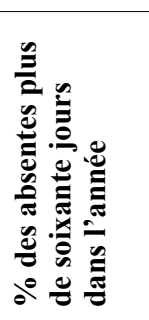 & 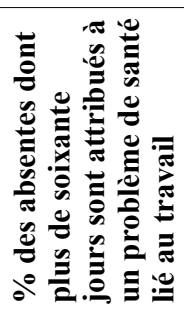 \\
\hline Belgique & 32 & 12 & 3 \\
\hline Danemark & 34 & 6 & 2,5 \\
\hline Allemagne & 26 & 4,6 & 2,5 \\
\hline Grèce & 13,5 & 2 & 1 \\
\hline Espagne & 12,5 & 5 & 2 \\
\hline France & 25 & 15 & 5 \\
\hline Italie & 28 & 8 & 1,5 \\
\hline Luxembourg & 29 & 2 & 0 \\
\hline Pays-Bas & 36 & 7 & 1,5 \\
\hline Autriche & 21 & 1 & 0,5 \\
\hline Portugal & 12 & 13 & 11 \\
\hline Finlande & 52,5 & 5,5 & 2 \\
\hline Suède & 32 & 12 & 8 \\
\hline Royaume-Uni & 23 & 6 & 3 \\
\hline Norvège & 31 & 11 & 3 \\
\hline
\end{tabular}

Source: European working condition survey 2005, European Working Condition Observatory, European Foundation for the Improvement of Living and Working Conditions.

Tableau 7bis : Indicateurs comparés de santé au travail en $\%$

\begin{tabular}{|c|c|c|c|c|c|c|}
\hline & 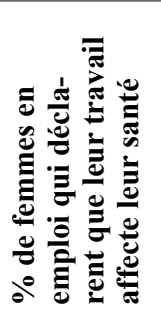 & 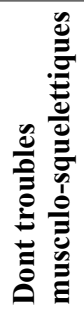 & 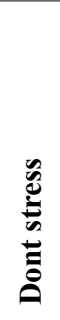 & 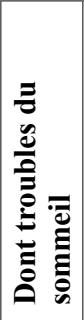 & 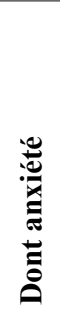 & 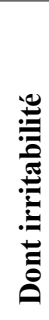 \\
\hline Belgique & 28 & 16 & 22 & 10 & 8 & 12 \\
\hline Danemark & 44 & 32 & 27 & 10 & 3 & 15 \\
\hline Allemagne & 20 & 12 & 13 & 3 & 0,5 & 5 \\
\hline Grèce & 62 & 43 & 53 & 14 & 30 & 22 \\
\hline Espagne & 28 & 21 & 18 & 6 & 8 & 10 \\
\hline France & 26 & 20 & 20 & 9 & 11 & 11 \\
\hline Italie & 37 & 22 & 25 & 9 & 15 & 13 \\
\hline Luxembourg & 35 & 25 & 29 & 14 & 13 & 17 \\
\hline Pays-Bas & 20 & 14 & 13 & 6 & 2 & 6 \\
\hline Autriche & 30 & 20 & 17 & 5 & 1 & 8 \\
\hline Portugal & 37 & 27 & 25 & 11 & 13 & 15 \\
\hline Finlande & 46 & 37 & 26 & 14 & 7 & 14 \\
\hline Suède & 57 & 42 & 41 & 20 & 18 & 15 \\
\hline Royaume-Uni & 18 & 8 & 11 & 6 & 6 & 6 \\
\hline Norvège & 48 & 33 & 30 & 11 & 12 & 14 \\
\hline
\end{tabular}

Source: voir tableau 7
Du côté des recherches socio-sanitaires menées en Suède, la situation n'apparaît pas moins préoccupante. La moitié des femmes actives en emploi déclarent être allé travailler au moins deux fois dans l'année en dépit de problèmes de santé (idem pour les hommes); $38 \%$ se disent exposées à des conflits avec leurs collègues de travail $(35 \%$ pour les hommes); $15 \%$ déclarent manquer de sommeil entre deux jours de travail (11\% des hommes); $55 \%$ se déclarent épuisées à la fin de leur semaine de travail (48\% pour les hommes); $46 \%$ déclarent effectuer un travail qui génère du stress mental (38\% des hommes); $18 \%$ n'ont pas l'envie d'aller travailler (17\% des hommes); $44 \%$ font état de troubles musculo-squelettiques ( $28 \%$ des hommes); $52 \%$ déclarent ne pas pouvoir choisir le moment de réalisation de leur travail (38\% des hommes); 18\% se déclarent exposées à la violence et à des menaces de violence dans le cadre de leur travail $(10 \%$ des hommes); tandis que $2,5 \%$ déclarent subir des agressions sexuelles de la part de leurs collègues et de leurs supérieurs, plusieurs fois durant l'année précédant l'enquête, $12,5 \%$ affirment subir des agressions fondées sur le genre de la part de leur milieu de travail et 7,5\% déclarent avoir subi plusieurs agressions à caractère sexuel de la part d'une personne tierce (18).

Les recherches socio-sanitaires apportent des éléments d'interprétation de la croissance des congés maladie chez les femmes, particulièrement dans la tranche d'âge des 20-39 ans. Leurs conclusions sont éclairantes. Tout d'abord, la séparation stricte dans le système de protection sociale suédois entre les congés pour prendre soin d'une enfant malade et les congés maladie proprement dits permet d'écarter l'hypothèse d'une prise de congés maladie des mères qui viserait la prise en charge des enfants souffrants. Par ailleurs, c'est la double charge, salariale et domestique notamment parentale, qui explique la fragilité psychologique et physique des femme en emploi (LundBerg et al., 2003).

D'après une étude récente sur le phénomène de burnout (19) en Suède (SANDMARK, 2007), avoir un premier enfant à un âge précoce, tout comme avoir d'autres enfants et en prendre la charge à titre principal sont des facteurs qui accroissent les risques de tomber en longue maladie. D'autres facteurs inter-

(18) Source: Arbetsmi betsmiljö ljöverket (www.av.se) och SCB (producent): Statistiska meddeland serie AM 68 (www. scb.se/AM050 0501). Le European working condition survey de 2005 fait état de fréquences d' "attention sexuelle indésirée», de menaces de violence physique au travail et de violence physique effective plus élevées en Suède que dans les autres pays européens (www.eurofound.europa.eu/ewco/surveys/ ewcs2005/results.htm).

(19) Le burnout se traduit notamment par une forte fragmentation du sommeil, voire une incapacité à dormir qui peut déboucher sur le suicide. On note à ce titre que le taux de suicide des femmes en Suède est supérieur à la moyenne de l'OCDE (source: Eco-Santé OCDE 2007). 
viennent, notamment la faible mobilité au travail et le temps partiel. La charge physique et mentale du travail constitue un autre facteur aggravant: les pressions professionnelles mentales et physiques se sont accrues y compris dans les services publics, notamment dans les services de prise en charge des personnes fragiles. Il apparaît que les femmes touchées par le burnout font état d'une charge de travail domestique supérieure à la moyenne, d'une plus grande propension à travailler «avec des gens » - métiers relationnels de la santé et du care notamment - et d'une soumission à une forte contrainte au travail. Il en va de même concernant l'inadaptation des compétences aux tâches effectuées: les périodes d'absence pour la naissance des enfants, puis pour les prendre en charge au foyer, ont un effet négatif sur les capacités d'intégration des femmes dans leur activité, notamment en rendant obsolètes leurs compétences dans un environnement professionnel en mutation rapide (STENLUND et al., 2007).

C'est donc bien un rôle domestique et parental encore exacerbé chez les femmes suédoises - malgré un contexte censé être favorable au partage des tâches domestiques - couplé à un environnement de travail certes adapté du point de vue temporel mais où règnent de fortes tensions, qui compromet leur santé.

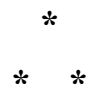

L'argument n'est pas ici d'affirmer de manière péremptoire qu'il existerait un «vrai» taux d'emploi des Suédoises que masque celui officiellement affiché, et qui consisterait en un réajustement à la baisse du taux officiel de manière à tenir compte des absences au travail, même si on l'a vu, elles sont considérables. Il s'agit plutôt de prendre conscience de la signification que couvre la mesure de l'activité et de l'emploi selon le contexte socio-économique dans lequel cette mesure est effectuée.

Ce que montrent d'abord les chiffres d'absence au travail c'est qu'il est tout aussi problématique de comparer des taux d'emploi que de comparer des taux d'activité. L'emploi prend en effet un contenu très différent selon les choix de société, reflet qualitatif du degré de décommodification atteint par un pays. C'est tout le problème des «cibles» de taux d'emploi définies par les accords de Lisbonne. L'artefact statistique de $60 \%$ de taux d'emploi chez les femmes en Europe peut correspondre à des réalités sociales très différentes, et ce, non seulement en termes de statuts d'emploi - temps plein versus temps partiel, CDI versus CDD - en termes de conditions d'emploi - niveau de qualification mobilisé - en termes de secteurs d'activité - services sociaux publics et parapublics versus services personnels et commerciaux privés - mais aussi en termes de degré de décommodification, c'est-à-dire d'autonomie des salariés par rapport aux forces du marché, autonomie dont le taux d'absence au travail permet de prendre la mesure avec une certaine pertinence, même si c'est imparfaitement. Il y a fort à parier que c'est sur ce taux d'absence, aussi bien sur son niveau global que sur sa composition, que vont se cristalliser les enjeux politiques des prochaines années. Ce qui rappelle une fois encore, que plus qu'une simple opportunité économique, Lisbonne est un défi politique et social: celui de la construction du plein emploi dans le respect d'une garantie d'autonomie des forces productives vis-à-vis du marché ... cette autonomie passant aussi par la préservation de l'état de santé des travailleurs.

La distribution des motifs d'absence au travail dans un pays donné est largement tributaire de ce que l'on y inclut. En France, les congés maladie supérieurs à un an tout comme les congés de formation de plus de trois mois, relèvent de l'inactivité, alors qu'en Suède tout congé maladie quelle que soit sa durée est comptabilisé comme congé d'une personne en emploi. Au Royaume-Uni, le nombre croissant de malades de longues durée est comptabilisé parmi les «inactifs en âge de travailler», une manière de les évacuer des chiffres du chômage (20).

Il y a donc bien à l'œuvre des critères et des conventions qui président à la délimitation des territoires de l'activité, de l'emploi et de l'inactivité dans chaque pays. Ainsi, ce n'est pas parce que la Suède présente une proportion plus importante de sa population en formation qu'elle forme plus sa population qu'un autre pays. Cette proportion montre que les personnes en formation sont considérées comme partie intégrante de la population en emploi, et non comme inactives, et qu'à ce titre elles échappent au risque professionnel que représente l'absence au travail. Il en va de même pour les autres formes d'absences.

Le fait de placer la frontière de la mise en emploi plus loin dans le territoire de l'inactivité que ne le font les autres pays, montre que la Suède accorde à l'emploi une définition extensive, intégrant des phases de la vie qui ne sont pas directement liées à situation d'emploi, mais qui participent plus ou moins directement à la reproduction de la force de travail. La décommodification pour raison familiale ou pour d'autres motifs (congés, congés maladie, formation) se conjugue ainsi à une logique de domestication des forces productives. Dans le cas du congé parental, le seul modèle vraiment reconnu en Suède étant celui $\mathrm{du}$ «citoyen-parent-travailleur» (VIELLE, 2006), l'assurance parentale se fonde sur la qualité de travailleur $\mathrm{du}$ parent et garantit un maintien presqu'intégral du revenu pendant les congés parentaux. On peut ainsi considérer que les $20 \%$ de femmes et les $13,5 \%$ d'hommes occupés et absents du travail sont les

(20) Francisco Vergara, «Emploi le mythe de la performance britannique», Le Monde 13 septembre 2005. 
représentants de cette logique de domestication des forces productives par laquelle ces dernières sont en quelque sorte convoquées dans l'accomplissement de tâches reproductives (21). Dans ce modèle, l'accès de tous à l'emploi et à la production, c'est-à-dire la généralisation de l'activation des forces reproductives, rend nécessaire l'implication simultanée de tous dans la reproduction. La mobilisation des forces productives dépend ainsi de cette implication. C'est cette articulation activation/domestication qui, en Suède, se trouve au cœur de la logique aggrégative famille-travail, et qui caractérise ce pays. Si la Suède esquive mieux que ses partenaires le «piège du genre»-défini comme la difficulté de s'émanciper des rôles sociaux assignés aux sexes masculin et féminin dans les sphères familiale et professionnelle (VIELLE, 2006) - en revanche cela ne l'empêche pas de développer paradoxalement une tendance simultanée à la domestication de ses forces productives: ce que l'on pourrait nommer le piège de la décommodification inachevée. Trois indices militent en faveur de cette lecture. Tout d'abord une forte participation des actifs hommes et femmes aux tâches domestiques: les individus ont le devoir de travailler et d'assumer leurs responsabilités parentales ou familiales. À ce titre il n'est pas sans intérêt de rappeler que la Suède a été jusque dans les années 1960 un modèle historique du travail domestique et salarié forcé pour les pères, encore appelé «paternité obligatoire». De fait les «maisons de travail», fermées en 1964, menaçaient les pères qui ne jouaient pas leur rôle de breadwinner, n'assumaient pas leurs responsabilités familiales et qui donnaient priorité à l'errance (du chômage notamment) plutôt qu'à la sécurité de leur famille (HoBson, 2003). Second indice, il existe beaucoup d'emplois dans les métiers liés à la reproduction - éducation, formation, santé, services sociaux - et la féminisation de ces métiers est extrêmement poussée. Enfin, troisième indice, si les femmes suédoises vivant en couple avec enfant(s) affichent un des taux d'activité les plus élevés au monde, on a vu que leur taux d'absence au travail, notamment pour maladie, est également parmi les plus élevés.

Le système social suédois présente l'indéniable intérêt de rendre visibles les absences au travail dans les statistiques d'emploi, ce que ne font pas la plupart des pays n'ayant pas poussé aussi loin l'émancipation à l'égard du marché du travail. Cette identification reflète la reconnaissance dont les absences des personnes en emploi font l'objet et traduit leur prise en charge financière dans le cadre d'une couverture

(21) Par contraste on peut avancer qu'un pays comme le Japon est porteur d'une logique presque inverse où il y a absorption, voire instrumentalisation des forces reproductives par les tâches de production. La différence des taux fertilité entre les deux pays est sans doute le reflet de ce qui sépare la perspective de recherche d'efficacité reproductive en Suède de celle de la recherche d'efficacité productive au Japon: 1,67 dans le premier contre 1,22 dans le second. sociale des risques professionnels. Mais pour autant les absences en question existent bel et bien, même si c'est dans un système qui les reconnaît juridiquement et qui les compense financièrement. Autrement dit reconnaissance statistique - qui est en fait surtout écho de l'inscription dans le droit social - et compensation financière, n'impliquent pas systématiquement prévention des risques sociaux et professionnels. La couverture du risque n'empêche pas manifestement ce dernier de se réaliser effectivement, et c'est ce que traduisent les chiffres alarmants en matière de congés pour cause de maladie.

En quoi peut-on parler d'inachèvement de la décommodification et en quoi cet inachèvement fait-il de la décommodification un piège?

La décommodification est, dans le cas suédois, inachevée parce qu'elle porte essentiellement sur l'émancipation des individus vis-à-vis du marché du travail, laissant l'émancipation par rapport au conjoint ou par rapport aux responsabilités familiales encore partielle.

En effet, une partie des fonctions familiales est «externalisée», ce qui génère un nombre important d'emplois dans des secteurs qui répondent à ces fonctions - d'où le poids des emplois de services sociaux - mais dans des conditions qui favorisent des pressions professionnelles importantes: temps partiel, horaires atypiques, qui encouragent un niveau élevé de ségrégation professionnelle. Une autre partie des fonctions familiales vient, pour des raisons conventionnelles - notamment la convention de l'enfant - alourdir l'emploi du temps domestique des femmes. Certes les femmes suédoises ne sont pas encouragées, comme c'est le cas dans nombre de pays, à utiliser leurs congés familiaux sous la forme d'une réduction de leur taux d'emploi. Certes le système de protection sociale n'aboutit pas à une spécialisation et une solidarité fonctionnelles entre, d'un côté les travailleurs affectés aux tâches de production et, de l'autre, les travailleurs affectés aux tâches de reproduction, même si une division genrée de ces fonctions existe bien. Pour autant les Suédoises se trouvent individuellement soumises à une double contrainte professionnelle et domestique qui génère des pathologies en nombre important. $\mathrm{Ce}$ sont là les dommages collatéraux d'une décommodification inachevée, dont l'ampleur des congés pris pour maladie constitue un indice.

Il est presque ironique de constater que c'est précisément l'émancipation par rapport au marché du travail qui légitime l'intégration parmi les risques faisant l'objet d'une couverture sociale, d'une part, des absences pour maladie - à l'émergence desquelles cette émancipation inachevée contribue ...-d'autre part des absences pour raisons familiales notamment pour s'occuper d'un enfant - absences qui viennent alourdir le budget-temps domestique des femmes. Quand la décommodification devient un piège. 
La perspective que nous prenons ici ne consiste pas pour autant à considérer les absences au travail comme un «simple» risque d'aléa moral qui s'accroîtrait notamment avec la générosité du système d'assurance sociale. En contrepoint de cette représentation de l'absence pour maladie comme forme d'opportunisme, nous pointons la nécessité d'aller plus avant dans la réduction de l'emprise des forces du marché sur la vie des individus et de faire de la décommodification un dispositif permettant de mieux affronter les risques liés au travail, notamment en œuvrant à leur minimisation. L'ampleur des absences pour maladie est un symptôme du caractère inabouti de la décommodification et non le syndrome de sa faillite.

\section{Bibliographie}

D'Addio A.C., Mira D'Ercole M. (2005), Trends and Determinants of Fertility Rates in OECD Countries: The Role of Policies, OECD Social, Employment and Migration Working Papers, Paris, OECD.

Adema W. (2004), «Rôle des congés parentaux dans les dispositifs de garde des jeunes enfants des pays de l'OCDE», Paris, Revue de l'OFCE, n' 90 .

Anxo D. (2007), "The gender division of labour in Europe: a life course perspective", in European Employment Strategy: Suggestion to Policy in Japan, Japan Institute for Labour Policy and Training, march, Tokyo.

Anxo D., Flood L., Kocoglu Y. (2002), «Offre de travail et répartition des activités domestiques au sein du couple: une comparaison entre la France et la Suède», Économie et Statistiques, ${ }^{\circ}$ 2, volume 352-353.

Blood R.0., Woolf D.M. (1960), Husbands and Wives, Illinois, The Free Press of Glencoe.

Bonato L., Lusine L. (2004), Work Absence in Europe, IMF Working Paper 04/193, Washington.

Brossolet C. (1993), «Spécialisation et déspécialisation sexuelle du travail: l'analyse économique», Revue Sociétés Contemporaines, $\mathrm{n}^{\circ} 16$, pp.145-163.

Daune-Richard A.-M. (2004), «Les femmes et la société salariale: France, Royaume-Uni, Suède», Travail et Emploi, n¹00, octobre, pp. 69-84.

Daune-Richard A.-M., Nyberg A. (2003), «Entre travail et famille: à propos de l'évolution du modèle suédois», in «L'État providence nordique», Revue française des affaires sociales, ${ }^{\circ} 4$, octobre-décembre.

ESPING-ANDERSEN G. (1997), «L'État protecteur à la fin du siècle - Les conséquences de l'évolution du marché du travail, de la famille et de la démographie», pp.65-85, in «Famille, Marché et Collectivité - Équité et efficience de la politique sociale», Études de politique sociale, $\mathrm{n}^{\circ} 21$, Paris, OCDE.

Hobson B. (2003), "The Interplay Between Indentities and Institutions - The Centrality of Swedish Women's Mobilization in Welfare State Expnasion and Retrenchment", pp.17-44, in C. le Grand and T. Tsukaguchi-le Grand (eds), Women in Japan and Sweden - Work and Family in Two Welfare Regimes, Stockholm Studies in Sociology ns.18, Almqvist \& Wiksell International, Stockholm.

KILKEY M. (2003), "Dual-earning couples in Europe: towards gender equality?" 1st Annual Conference of ESPAnet, The Network for European Social Policy Analysis, Copenhagen.

LALLEMENT M. (2002), «Régulation des temps sociaux en France et en Suède», Économies et Sociétés, $\mathrm{n}^{\circ} 22$, 8/2002, p.1349-1367.

LETABLIER M.-T. (2003), «Les politiques familiales des pays nordiques et leurs ajustements aux changements socio-économiques des années 1990 », in «L'État providence nordique», Revue française des affaires sociales, $\mathrm{n}^{\circ} 4$, octobre-décembre.

Lundberg U, Krantz G, Berntsson L. (2003), "Total workload, stress and muscle pain from a gender perspective", Journal of Sociological Medecine, Vol. 3, pp.245-254.

Marc C. ZaJdela H. (2004), «Emploi et politique familiale: doit-on s'inspirer du "modèle suédois"?», actes du colloque MATISSE : accès inégal à l'emploi et à la protection sociale, Paris, 16-17 septembre 2004.

MelKas H. AnKer R. (2003), "Towards Gender Equity in Japanese and Nordic Labour Markets: A Tale of Two Paths", International Labour Office, Geneva, InFocus Programme on Socio-Economic Security, July.

Meulders D. de Henau J. Maron L. O’Dorchai S. (2007), «Travail et maternité en Europe, conditions de travail et politiques publiques », Brussels Economic Review, vol. 50, $\mathrm{n}^{\circ} 1$ Spring.

Morgan Kimberly J. (2006), «Les politiques du temps de l'enfant en Europe occidentale: tendances et implications», Recherches et Prévisions, $\mathrm{n}^{\circ} 83$ mars, pp.29-43.

NyströM L., GAunt D. (1986), «Le modèle scandinave», in André Burguière et al. (eds), Histoire de la famille, pp.607-641, Paris, Armand Colin.

OCDE, (2007), Economic Survey of Norway, Paris.

OCDE (2008), Economic Survey of Sweden, Paris.

Ogura K. (2004), "Annual Paid Leave in Japan”, Japan Labour Review, Vol. $1 \mathrm{~N}^{\circ} 2$, Japan Institute for Labour Policy and Training, Spring.

Presser H.B., Gornick J.C. (2006), "The female Share of weekend employment: a study of 16 countries", Monthly Labor review, August, pp. 41-53, US Department of Labour.

RAE D. (2005), "How to reduce sickness absences in Sweden: lessons from international experience", OCDE Economic Department Working Paper $n^{\circ} 442$.

SANDMARK H. (2007), "Work and family: associations with long-term sick-listing in Swedish women - a case-control study", BioMedical Central Public Health, Vol.7, p. 287.

Stenlund T., Ahigren C. (2007), "Patients with burnout in relation to gender and a general population", Scandinavian Journal of Public Health, Vol. 35, №. 5, pp. 516523.

VIELLE P. (2006), La représentation des femmes et la construction des rôles parentaux - Une approche comparative du droit de la Sécurité sociale, document ronéotypé, université catholique de Louvain.

WeHner C., Abrahamson P. (2003), "Family and/or work in Europe", 1st Annual Conference of ESPAnet, The Network for European Social Policy Analysis, Copenhagen. 